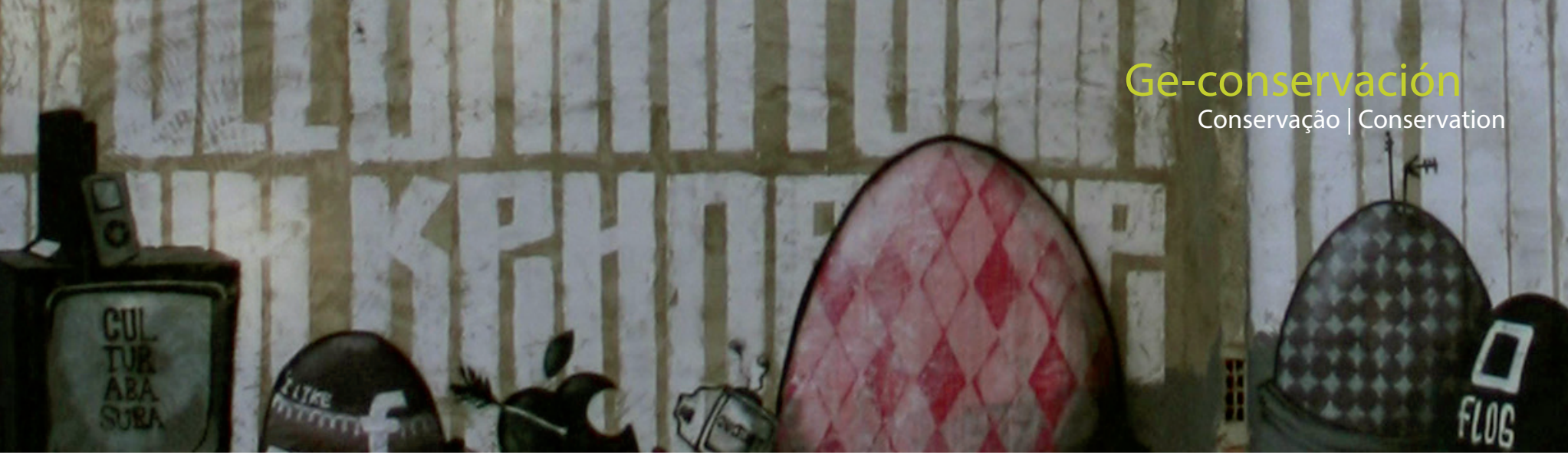

\title{
La conservación del arte urbano. Dilemas éticos y profesionales
}

\section{Carlota Santabárbara Morera}

Resumen: La conservación del arte urbano no pasa sólo por la conservación de la imagen y la materia que la soporta, sino también por la conservación de toda una serie de factores ambientales y de contexto que la condicionan para pervivir y evolucionar, en un ambiente urbano. Se plantea una metodología de estudio para la conservación basada en una perspectiva ética.

Palabras clave: restauración, autenticidad, arte urbano, conservación, intencionalidad

\section{The conservation of urban art, ethical and professional dilemmas}

Abstract: The conservation of street art is not only the conservation of the image and the matter which is the support of it. But also the conservation of a whole host of environmental and contextual factors, rather condition to survive and develop, in an urban environment.. The methodology of study showed is based in an ethics perspective.

Key words: restoration, authenticity, street art, conservation, purpose

\section{Introducción. Objetivos y metodología}

La conservación del arte urbano constituye un campo incipiente dentro del ámbito de la conservaciónrestauración. Existen ya algunos ejemplos donde el interés social o institucional ha llevado a la documentación, restauración e incluso al arranque y musealización de algunas obras, promovidas, en la mayoría de los casos, por intereses turísticos y económicos.

El objetivo de este trabajo es plantear preguntas que arrojen luz sobre los límites de lo que es lícito a la hora de intervenir en las manifestaciones artísticas urbanas y con qué criterios éticos. Para ello, es aconsejable partir, en primer lugar, de la comprensión y definición del objeto de estudio.

Se propone analizar el arte urbano como arte actual, popular, que se manifiesta de un modo completamente libre a nivel creativo, tanto desde el punto de vista técnico como material y que se apropia del espacio público utilizándolo como contexto de comunicación e instrumento de expresión. Es necesario revisar el nivel conceptual de las obras para diferenciar claramente cómo afecta, por un lado, a las manifestaciones creadas de forma espontánea, furtiva y libre, y por otro, a las intervenciones que son comisionadas desde el ámbito institucional, ya que el motivo de su génesis determina su razón de ser y por lo tanto, su identidad.

La metodología propuesta traza un estudio en paralelo con otras obras conocidas ya conservadas o restauradas de Arte Contemporáneo, sobre las que se han podido plantear estrategias que permiten un análisis intelectual previo y en las que se observan similitudes conceptuales o materiales similares a algunas de arte urbano. 


\section{Arte furtivo y arte de encargo}

En primer lugar es imprescindible conocer y describir la naturaleza del arte urbano para poder plantear estrategias y metodologías de conservación. Para hacer una descripción de los valores que definen una obra de arte urbano se deben perfilar primeramente sus características más genuinas. En un primer grupo estarían las obras en las que se busca identificar el impulso creativo con su forma de creación, como es el caso de los writers, que son referencia y paradigma de ilegalidad. Como ejemplos de esta manera de actuar se encuentra Kirax, artistas más activistas como Vermibus, que interviene sobre las fotografías publicitarias de las marquesinas, o Banksy, el más mediático, que realiza sus pinturas de manera provocadora en las calles de todo el mundo y siempre con un mensaje político. En estos casos, plantear la conservación de expresiones artísticas urbanas resulta bastante contradictorio, porque se trata de un tipo de intervenciones anónimas, con un trasfondo que invita a la reflexión y a la reacción. Sin embargo, con ellas se ha producido un proceso de transformación social, donde lo que en origen fue un fenómeno denostado y rechazado, por incomprendido y por su carácter ilegal o vandálico, ahora se recibe como una intervención estética que da valor añadido a las ciudades, genera un creciente interés social, cultural y turístico, aportando un atractivo carácter artístico al ámbito urbano. Artistas como ROA, Borondo o Blu viajan por toda Europa dejando testimonio de su creatividad, llenando las calles de murales y generando la expectativa de poder encontrar alguna de sus intervenciones en cualquier lugar recóndito. De este modo, se ha ido generando un atractivo que despierta en la sociedad el deseo y la necesidad de pertenencia y permanencia de obras urbanas. Sin embargo, esa necesidad de posesión y deseo de perpetuarlas se ha producido a posteriori, por un proceso de reconocimiento social, ya que no se crearon con la intención explícita de perdurar en el tiempo. Por lo tanto, el debate que se genera en torno a la conservación o no del graffiti y el arte urbano parte también de un reconocimiento de su valor artístico, en cuanto a objeto de galería.

En segundo lugar, estarían las obras cuyo interés se centra en el concepto mismo de su representación. Restaurar apresuradamente por el mero hecho de conservar una obra, sin analizar los motivos por los que se hace, llevaría a cometer el mismo error que se cometió al restaurar y sustituir la esquina de grasa en caja de cartón de Josep Beuys (Fettecke in Kartonschachtel), y que, al ser adquirida por el Stedelijk Museum de Amsterdam en 1972, se consideró que no sólo se debía conservar, sino también restaurar. Cuando el proceso de deterioro de las grasas animales presentes en la obra provocaron la descomposición de los ácidos grasos y comenzó a desprender un olor a putrefacción muy desagradable, se decidió sustituir por otro material que se asemejara estéticamente, pero con una composición diferente. Entre los materiales elegidos se utilizó mayoritariamente cera de abejas (que era mucho más estable al proceso de degradación) pero la sorpresa del restaurador y del museo fue cuando el propio artista consideró que esa restauración era completamente errónea y la obra había perdido su valor. Según el propio Beuys, la restauración no era una opción viable, puesto que el proceso de deterioro formaba parte de la evolución de la propia obra.

Este ejemplo nos sirve para ilustrar lo que ocurre con tantas obras de arte que son restauradas y conservadas manteniendo su estética aparente, pero olvidando que su importancia radica en otra parte, al igual que puede llegar a ocurrir con el arte urbano, donde la voluntad argumentada del artista debe ser conocida con respecto a las expectativas de conservación y restauración.

En tercer lugar, no sólo abordaremos el caso de las obras de arte urbano espontáneas, sino que también nos atañe, y cada vez más, otro tipo de intervenciones artísticas comisionadas, enmarcadas dentro de un festival o proyecto institucional. Estas intervenciones ofrecen unas posibilidades de conservación quizá más reconocibles, ya que los muros son ofrecidos como soportes artísticos y las obras proceden de encargos. Hoy en día, son muchos los ayuntamientos que organizan festivales de arte urbano convocando a artistas para realizar intervenciones en la calle que mejoran estéticamente las zonas degradadas de las ciudades, como es el Under Pressure de Toronto, el ONO'U Battle en Tahití, o el Nuart en Stavanger, en Noruega. En España, los más conocidos son el Festival Asalto en Zaragoza y Open Walls de Barcelona.

De hecho, cada vez son más difusas las transiciones entre el arte, la arquitectura y la planificación de la ciudad, en cuanto a diseño urbano y sus usos. Y es en esta línea tan sutil donde las políticas de gentrificación manipulan el arte urbano para apropiarse del espacio público y con una apariencia amable y silenciosa llegar a cambiar el aspecto de las ciudades, al propiciar la especulación inmobiliaria.

Sin duda, la expresión artística cambia radicalmente en cuanto a contenido, intención y modus operandi cuando se trata de intervenciones realizadas bajo demanda, y aunque siempre se realizan en el espacio público, sus características al servicio de intereses de terceras personas lo singulariza y diferencia del resto de intervenciones de arte urbano independiente. La periodista especializada en cultura Paloma Fidalgo lo define como "la versión aseada del grafiti" y es que, cada vez más, las instituciones, a través de festivales y convocatorias artísticas, están propiciando la práctica de un "muralismo contemporáneo" políticamente correcto. Se trata de creaciones cuyo origen e intención es notablemente diferente a las manifestaciones conocidas dentro del Postgraffiti, que ahora se instalan como obras decorativas y tal vez con un merchandising excesivo. Por lo tanto, la intención con la que son creadas no solo es legal, sino que se ha institucionalizado $y$, por tanto, es susceptible de ser conservado y restaurado sin que su concepto se vea alterado. 


\section{Los límites de lo efímero}

Ante la posibilidad de conservar o no las manifestaciones artísticas desarrolladas en el ámbito urbano, una de las premisas fundamentales desde la que hay que partir es la de conocer qué es lo que se considera fundamental en ellas. Si su valor radica en la imagen creada, en la experiencia técnica o en la interacción social de un lugar determinado. Para garantizar la permanencia de su autenticidad como manifestación artística habría que conocer, en primer lugar, su identidad creativa, ya que tal vez la intención de conservar y "congelar" una creación se puede contradecir con la razón por la que fue concebida.

En el arte urbano, las intervenciones tienen tendencia a transformarse con el paso del tiempo e, incluso, a ser borradas de un modo natural, tal es el caso, por ejemplo, de las intervenciones que usan el papel, como es el caso de las producciones de Andrea Btoy, que gusta también de elegir soportes en los que se nota el deterioro y así las alteraciones aportan un valor estético en la materia degradada.

En este grupo es interesante la reposición de una obra del artista parisino Xavier Prou, Blek le rat, conocida como La Madonna. Situada en la ciudad alemana de Lepzig, fue creada como efímera, se perdió y posteriormente se recuperó. En 2012 se eliminaron los carteles publicitarios que habían cubierto la imagen durante más de dos décadas, se limpió y fue repintada por el propio artista, protegiéndose con un metacrilato y colocándose una cartela explicando la historia de la obra y cómo estaba dedicada a la que fue su esposa. En este caso, es importante no sólo la restauración y protección, sino también la difusión del valor de una obra que es reconocida y valorada socialmente como tal. Se trata de un ejemplo no sólo de conservación, sino de recuperación de una imagen icónica, a partir de la reproducción con la plantilla original y por el propio artista.

Llegados a este punto, se tendría que plantear el valor de este tipo de obras, no tanto por su autenticidad material, sino por su valor simbólico, que es lo que la hace que sea digna de conservarse y reproducirse. Sin lugar a duda, tal y como preconizaba el filósofo alemán Walter Benjamin, estamos en la era postaurática, en la que la imagen es reproducible y se desprende de su aura de obra única.

En esta misma línea, podemos mencionar cómo en 2015, se realizaron dibujos con pintura plástica sobre el mobiliario urbano de las marquesinas del tranvía en el Festival Asalto de Zaragoza. Sin duda todas estas creaciones fueron concebidas con la certeza de ser efímeras, y se podría decir que de forma performática, procesual, de interacción con el medio, con la comunidad, para dialogar, provocar, contar, o tan sólo impactar en un momento espacio-temporal concreto, a lo que hay que añadir la degradación prematura que sufrieron por estar realizadas sobre cristal. [Figuras 1 y 2].

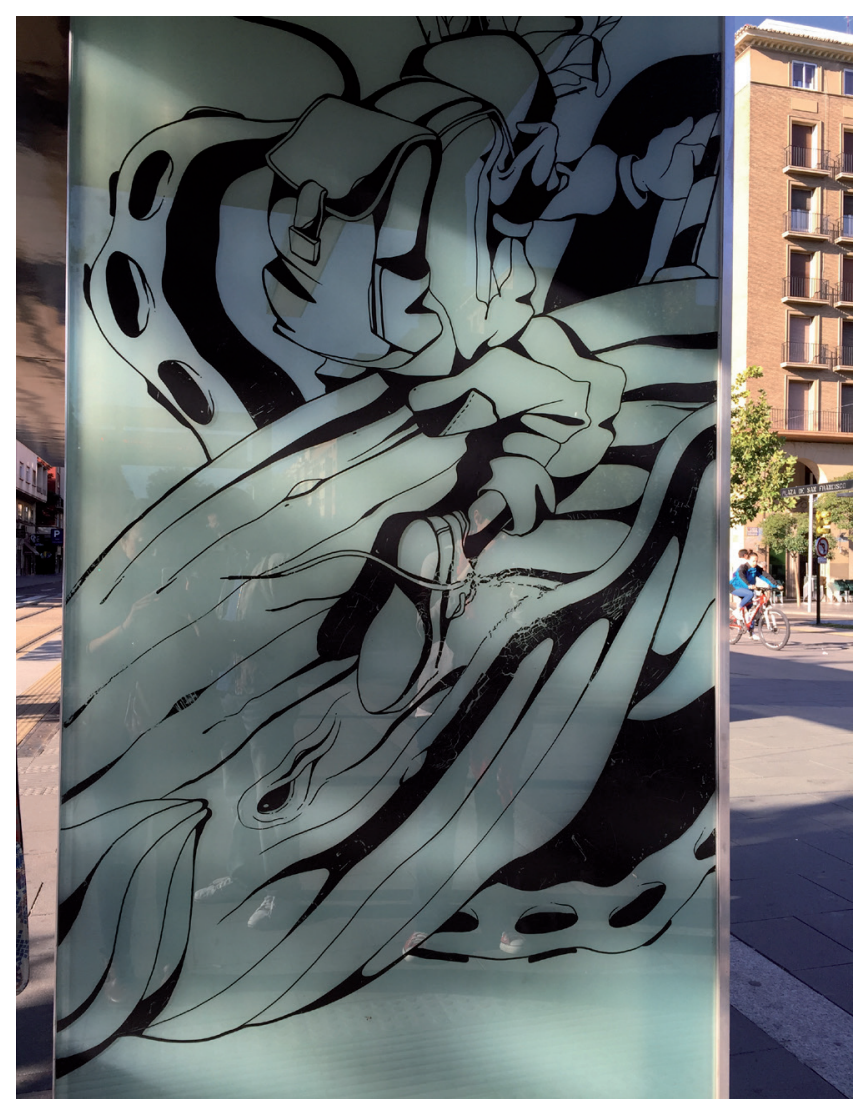

Figura 1. Festival Asalto de Zaragoza. Pintura acrílica negra sobre vidrio. Foto de la autora

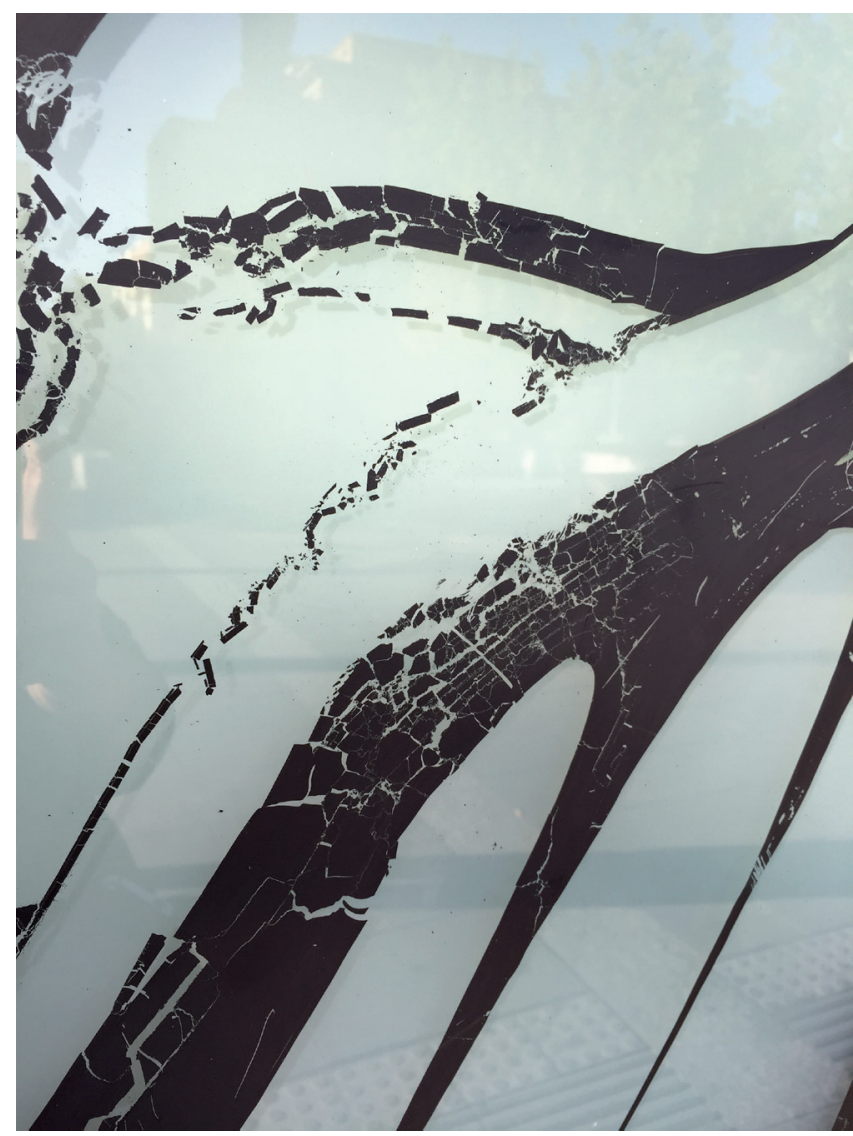

Figura 2. Festival Asalto de Zaragoza. Pintura acrílica negra sobre vidrio. Detalle de degradación. Foto de la autora. 
El concepto de arte efímero, que nace con una voluntad explícita de no ser conservado, existe también en el panorama del arte contemporáneo, donde hay numerosos ejemplos de obras creadas con una intención efímera y cuya conservación o restauración ha llegado a ser motivo de polémica. Tal es el caso de instalaciones de corte conceptual, como es el caso de la obra de Zoe Leonard Strange Fruit (for David), creada en 1993, consistente en una instalación de 302 cáscaras de diferentes frutas que fueron vaciadas. Se trataba de pieles de diferentes frutas que una vez secas, fueron cerradas con zurcidos, decorándolas con botones, cremalleras o suturas de hilo. [Figura 3].

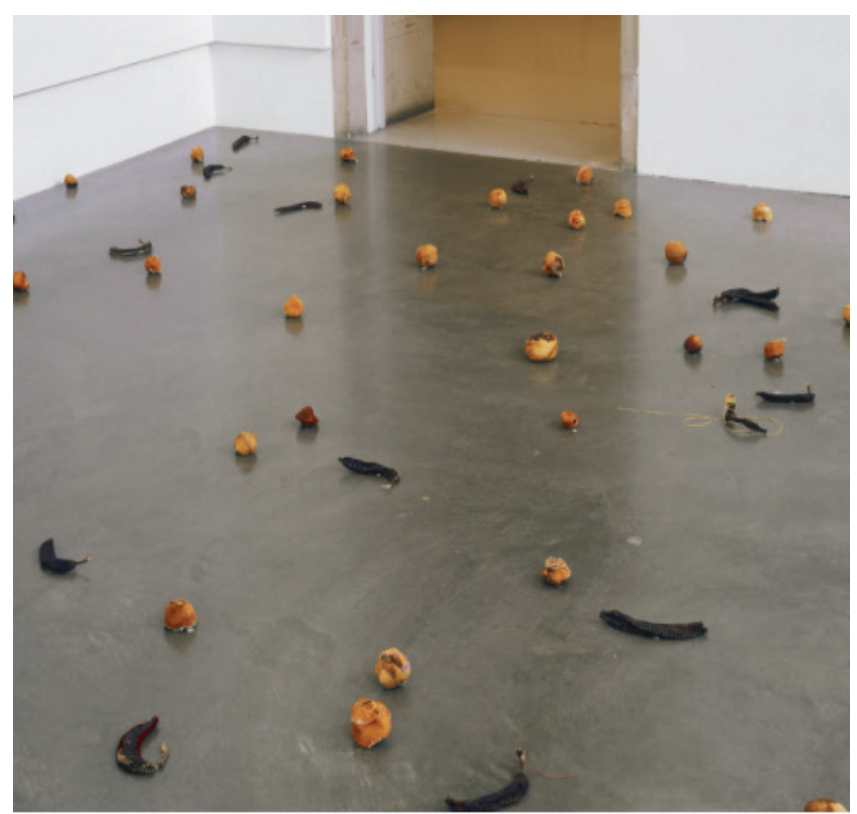

Figura 3. Zoe Leonard. Strange Fruit. Philadelphia Museum of Art. Purchased with funds contributed by the Dietrich Foundation and with the partial gift of the artist and the Paula Cooper Gallery, 1998. http://www.philamuseum.org/collections/ permanent/92277.html

Esta obra tenía una intención explícita al mostrar su degeneración material, dejando que las pieles de fruta se pudrieran hasta que dejaran de existir. Se trata de la representación simbólica del luto después de la muerte de un amigo, reflejando así el tema de la transitoriedad de la vida, como ya se hacía en el barroco con las vanitas. El problema surgió cuando fue adquirida por el Philadelphia Museum of Art, que tenía la intención de conservarla, pero respetando el derecho moral a decidir del autor, hubo que mantener la intención original con la que fue creada y finalmente se dejó autoconsumir hasta desaparecer. Este no deja de ser un ejemplo de cómo el arte contemporáneo va más allá de la mera representación formal, involucrando conceptos semióticos que es necesario conocer y respetar, al igual que ocurre con el arte urbano.

El propio proceso creativo, en la parte que afecta a la técnica elegida, va a definir su expectativa de supervivencia y qué aspecto de la obra va más allá de lo puramente pictórico; tal es el caso de Borondo, que realiza dibujos sustractivos por medio de rayados sobre los cristales de escaparates en desuso, pintados de blanco, creando un efecto de claroscuro muy impactante, pero que claramente están abocados a desaparecer en cuanto el local sea reabierto y los cristales deban lucir transparentes y limpios para un nuevo uso o según el gusto del nuevo propietario. [Figura 4].

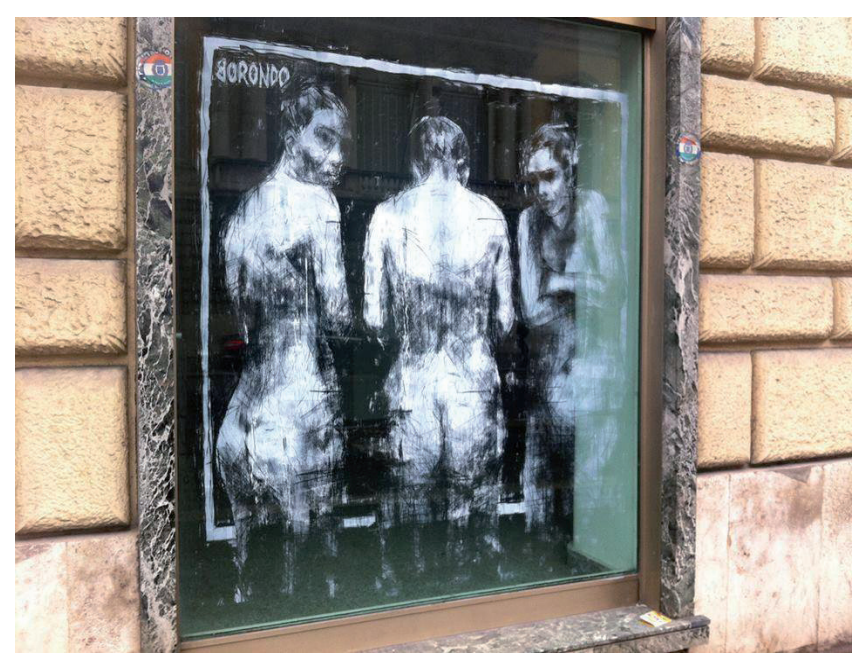

Figura 4. Borondo. en Via Nazionale. Roma. Foto de la autora

Por otro lado, es importante tener en cuenta la voluntad de los artistas por mantener el anonimato y no ser localizados, para consultarles si su obra es efímera o no. La inmediatez de respuesta a un hecho social o el clamor reivindicativo planteado desde un punto de vista político-social, propone una serie de cuestiones por resolver: desde la necesidad de permanencia y conservación, hasta el respeto a un devenir temporal y procesual propio de un arte efímero vinculado a un momento concreto que, a su vez, está inscrito en un contexto socio-cultural determinado y por lo tanto, exento de la posibilidad de ser patrimonializado.

\section{Paralelismo metodológico}

Si nos centramos en la estrategia conservativa, y desde el punto de vista documental, se podría adoptar el método de trabajo planteado por el grupo de investigación de la Foundation for the Conservation of Modern Art de Ámsterdam, que ya en 1993 propuso un modelo para guiar al proceso de decisión que precede a la restauración. ${ }^{1}$ En relación al método mencionado se trata de un esquema que ofrece una discusión preliminar para abordar el camino que llevará al razonamiento y a la toma de decisiones. Este modelo plantea que, en primer lugar, es necesario recopilar toda la información referente a la obra, tal como las noticias técnicas e históricas, así como averiguar el significado y la poética de la intencionalidad artística con la que fue creada, para, a continuación, detallar el estado de conservación y, posteriormente, determinar si la alteración del material 
puede llegar a cambiar el sentido original de la obra. Del mismo modo, en este proceso de investigación, su estado de conservación ha de compararse con el resto de obras de esa misma corriente artística, autor, estilo y mensaje específico, determinando que, sólo si el deterioro es de tal envergadura como para hacer ininteligible el trabajo, se puede programar la intervención.

Desde la perspectiva legal, y teniendo en cuenta el conflicto de intereses presente en las obras de arte urbano, se debe plantear la doble condición observada en estas obras: por un lado, la propiedad intelectual, que es del artista, y por otro, la propiedad de la obra a nivel de pertenencia, que es del propietario de la casa que contiene los muros intervenidos, en el caso de que se trate de una fachada. Ambas propiedades entran en conflicto desde el momento en el que una obra es realizada apropiándose del espacio urbano; el artista conserva el "derecho moral" mientras que el dueño del soporte físico tiene el derecho legal de su propiedad. Aunque la obra deja de pertenecer al artista, desde el momento que la entrega a la colectividad, al espacio urbano, su "derecho moral" impide que se especule con ella, sobre todo si nunca tuvo la intención de comercializarla o de entrar en los canales establecidos del mercado. En este caso, se crearía un conflicto que llevaría a recurrir a la jurisprudencia y analizar casos similares.

Desde el punto de vista ético, el abanico de opciones, así como todas sus variables, ha de ser examinado mediante la identificación de las posibles soluciones técnicas, distinguiendo lo que es factible desde el punto de vista práctico de lo que es adecuado desde el punto de vista ético. Basándose en esta premisa, se debe hacer una elección de compromiso entre las exigencias expresadas, identificando la solución que sea considerada como un mal menor, sin olvidar los problemas prácticos, la unicidad de la obra o los aspectos legales. Por lo tanto, el proyecto de restauración vendría a ser un instrumento de reflexión, que garantizaría la unidad de método y el conocimiento profundo de la obra, y en consecuencia, el restaurador actuaría como un mediador entre la conservación de la parte material de la obra y su parte intelectual.

\section{El margen de los criterios de intervención}

En la conservación de obras en las que intervienen y tienen opinión muchas personas, hay que partir de la certeza de que se pueden dar opciones extremas de conservación, por la diferencia de puntos de vista y objetivos que nada tienen que ver con la restauración, pero en los que ésta se convierte en una opción necesaria justificada fundamentalmente por exposiciones, proyectos comisariados o decisiones del dueño del soporte. Entre las opciones están las intervenciones del artista, los arranques y la musealización.
De la mano del propio artista, se pueden dar situaciones en las que éste quiere realizar una modificación más allá de su conservación, llegando a crear una obra nueva, traspasando los límites de la conservación y alterando el original, el cual habría dejado de existir. Por ello, la decisión debería de tomarse siempre de acuerdo con el propietario del soporte.

También se suele dar, demasiado a menudo en el arte urbano, la posibilidad de decidir el arranque de la pintura del muro para su traslado y conservación en soportes inertes, con el consiguiente cambio de contexto. La obra inmueble se convertiría entonces en mueble y variaría también su tipología. Este hecho, que paraliza la degradación material a la que están expuestas las obras pintadas a la intemperie, distorsiona por completo la propia concepción como arte público y urbano, no sólo en su sentido original, sino especialmente en su relación con el entorno, que resulta alterado en su efecto visual y emocional, así como tantos otros valores que son de gran importancia en la percepción experiencial de la obra urbana.

Es importante por ello tener en cuenta los valores de la obra en su restauración y no al revés, porque si no, se crearía una falsa historia estética, que llevaría a procesos tales como el de la realización de réplicas. Este tipo de actuaciones, aunque hicieran referencia al emplazamiento inicial, podrían desencadenar la musealización de las copias y la pérdida del original.

Es necesario, por tanto, desarrollar una reflexión preliminar a la restauración de una obra de arte urbano, sobre todo en cuanto a las opciones de acción, dado que es el artista el que determina la naturaleza perenne, transitoria o efímera de su obra, los materiales elegidos y cómo usarlos, de acuerdo a unos criterios estéticos específicos. Esta cuestión adquiere cierta complejidad cuando la recopilación de datos técnicos debe hacerse a través de sus herederos, sobre los que recaería el derecho moral del artista tras su fallecimiento, ya que sería un intermediario el que tendría que interpretar la voluntad del artista.

Es interesante observar cómo la percepción de una obra y los valores a conservar varían de una persona a otra, incluso dentro del ámbito profesional e institucional. Por ello, es necesario un programa conjunto de investigación y documentación que permita catalogar las obras y definir los principios generales de las intervenciones, un requisito que se ha desarrollado en el campo de la restauración del arte contemporáneo en la International Network for the Conservation of Contemporary Arte $(\text { INCCA })^{3}$, en torno a los procesos y protocolos de recopilación de información directa de los artistas y de su entorno, lo que permite la elección de unos criterios de intervención objetivos y universales.

“La restauración de obras de arte contemporáneas es muy diferente a la de las obras Ilamadas tradicionales, 
pues carece de la tradición de una práctica secular y, por supuesto, de una teorización propia" (Martínez Justicia, 2000: 443), por ello, los retos que se presentan ante la restauración de arte urbano requieren de planteamientos también diferentes.

Hoy en día, el arte se sirve de un tipo de comunicación desigual, ya no se trata de la mera representación formal, sino que va más allá, transmite, comunica, provoca y sobre todo crea experiencias. Por ello, lo importante es averiguar dónde reside la autenticidad de esas manifestaciones para evitar que dejen de tener sentido en el ámbito urbano.

En las teorías conservativas alemanas (Hiltrud Schinzel, 2003), se plantea la recuperación del Kunstwollen (la voluntad artística) expuesta ya por el historiador del arte austrohúngaro Alois Riegl a finales del siglo XIX (18581905), superando la restauración crítica. Del mismo modo la fenomenología del filósofo moravo Edmund Husserl (1859-1938) nos puede acercar más a un planteamiento conservativo basado en la experiencia, la emoción y las sensaciones que nos ofrece el arte urbano, buscando la recuperación de la intencionalidad, rescatando el pensamiento y la voluntad del artista y diferenciando entre lo que es la autenticidad y la originalidad. Debe existir la consciencia de la intención artística como valor fundamental a conservar, por lo que es de vital importancia averiguar por qué y para qué fue creada la obra de arte urbano, para así poder conservarla.

Por lo tanto, podemos afirmar que, si la restauración consiste en preservar la autenticidad del arte, la misión del conservador-restaurador será la de recuperar la intención con la que se creó, la voluntad de producir experiencias y emociones y en definitiva, recuperar el sentido que provocó su creación original. Estas serán las bases en las que se marquen las pautas de la actuación a realizar.

\section{La restauración dinámica o cómo conservar el cambio}

En la concepción occidental de la conservación, existe un gran empeño por la conservación de la materia, pero tal y como afirmaba el filósofo Theodor W. Adorno, el arte es algo más que materia y forma, ya que observó cómo en el arte, al igual que en la música, se necesita de un proceso temporal para su desarrollo, es decir, un carácter social en el que circunscribirse para poder existir. Resulta de gran clarividencia la diferencia que realiza el filósofo Massimo Carboni, desde un punto de vista teórico y filosófico, entre el arte tradicional y el arte contemporáneo y que sería pertinente recuperar aquí para analizar el arte urbano, lo que él Ilama: "la obra de la contingencia, en la que se alude, en el acto mismo de presentarse, a su propia desaparición, a una intencionada e irremediable caducidad." (Carboni, 2014:9)
De este modo, el arte urbano implica una percepción estética colectiva y esa experiencia comunitaria viene vinculada casi siempre a un momento concreto político-social del que no puede ser separado sin correr el peligro de aparecer descontextualizado, y por lo tanto, ser claramente transitorio y efímero. En este sentido y en relación a la Teoría estética de Adorno (Adorno, 1975), Carboni cuestiona cómo "la idea de duración de la obra es modelada sobre su categoría de posesión"(Carboni, 2014.12), es decir, presenta como la creación contemporánea va perdiendo paulatinamente su carácter de objeto y tiende a convertirse en un gesto, una acción o un proceso, por lo que podríamos ponerla en paralelo con la danza o la performance, es decir, un arte sin objeto físico como soporte, lo que nos llevaría a cuestionarnos hasta qué punto se puede conservar una acción. Para el filósofo italiano, la memoria es la metodología correcta y paradójica para respetar su legado estético-cultural y conservarlo para el futuro. En este sentido, cuando el historiador y crítico de arte Cesare Brandi hablaba del máximo respeto al original, hoy en día se debe entender "lo original" o "lo auténtico" ya no como un valor que reside en la materia, sino en la idea creativa, en la expresión artística o en la experiencia sensorial, es decir, en el proyecto o en el concepto artístico en sí mismo.

Por ello, y en relación a la conservación del arte urbano, tal vez lo más adecuado sería admitir que se debe conservar el cambio como una metodología que implique dinamismo en su propia definición. Precisamente, en la Conferencia de Nara, (Japón, 1994) se estableció una teoría que se inclinaba hacia una restauración dinámica, en lugar de una conservación estática, que llegase a congelar el objeto. Sin duda, conservar el cambio es quizá el reto más interesante para los restauradores de arte urbano de hoy en día. Por lo tanto, podemos aseverar que el arte ha evolucionado desde mitad del siglo XX partiendo de una representación material que constaba de un soporte físico y la representación de una imagen, hacia un arte basado en la percepción de un nuevo lenguaje conceptual, donde evidentemente continúa existiendo la materia como soporte de una imagen, pero esta materia evoluciona adquiriendo nuevos significados, sobre todo en el arte urbano, donde los aspectos políticos, sociales y culturales se entremezclan.

\section{Arrancar o conservar "in situ"}

Una vez analizado el objeto de estudio, y su definición, así como los valores que engloba, es obligado hacer las siguientes preguntas: ¿se debe conservar? ¿cómo se debe llevar a cabo ese proceso?

En primer lugar es necesario hacer un análisis cuidadoso a nivel físico, es decir, de los materiales que lo componen, y en segundo lugar averiguar la 
intencionalidad artística para conformar una propuesta conservativa a través de la perspectiva aportada por las distintas profesiones: los conservadores, historiadores y críticos, científicos y artistas, para evaluar sobre todo la necesidad de la intervención y las posibilidades operativas diferentes.

Cabe mencionar que existen ya bastantes ejemplos de arranques de graffitis y de murales urbanos que han sido comercializados con la finalidad de especular con creaciones que no pertenecen a las galerías o casas de subastas, pero que se apropian de ellas para beneficio propio. Es el caso de la subasta de los murales expoliados de Banksy procedentes de diferentes ciudades de Alemania e Inglaterra, obras que se vendieron por más de tres millones de euros sin la autorización ni la consulta al artista. Estas obras fueron expuestas sobre estructuras metálicas en el lujoso hotel ME de Londres, en una muestra llamada: Banksy robado, que precedió a una subasta organizada con fines benéficos. Pero estas exposiciones, con una expectativa claramente mercantilista, no son casos aislados ni nuevos, como el caso ya conocido de la exposición organizada en 1979 en Roma The Fabulous Five: calligraffitti di Frederick Brathwaite e Lee George Quinones, organizada en la Galleria La Medusa. Pero lo que llama más la atención ya no es sólo la incorporación del street art a nivel comercial, sino también la actitud de la institución museística, desde donde se intenta patrimonializar sin justificación alguna un tipo de arte que no debe ser introducido en un espacio museístico, sin un razonamiento previo. Este fue el caso en 1984 de la exposición Arte di frontiera. New York Graffiti, realizada en la Galleria Comunale d'Arte Moderna di Bologna, o Street Art. Banksy \& Co. L'arte allo stato urbano llevada a cabo este mismo 2016, en Bolonia (Italia), en la cual, con la idea de mostrar la evolución del graffiti y arte urbano se exponían piezas de los años setenta y ochenta de Nueva York y proponía una comparativa con Italia. Para esta ocasión se expusieron murales del artista urbano Blu, que habían sido arrancados de sus fachadas y musealizados, aparentemente sin su permiso. Sin duda se trata de la descontextualización de unas obras generadas por y para un ambiente determinado, en las que la imagen permanece, pero desaparece su identidad y su relación con el entorno que la generó.

Estas manifestaciones artísticas deberían permanecer in situ en el lugar donde fueron creadas. No se puede olvidar que el arte urbano, el graffiti y el muralismo comisionado o no, no puede desvincularse de su lugar de creación ya que éste forma parte de su propia definición como lenguaje de comunicación social en un contexto urbano determinado. Además, cabe señalar que la originalidad es un valor que tiene diferentes acepciones según el ámbito de su percepción. Por ejemplo, en el mercado del arte, la originalidad es un valor determinante para otorgar una cotización en su tasación económica, sin embargo para los visitantes de un museo es garantía de experiencias estéticas auténticas. A esto hay que añadir que desde el punto de vista de la conservación-restauración, dentro de su concepción de la originalidad del arte, la originalidad se basa en que un objeto ha sido realizado sin falsificaciones, tanto en el carácter material, como técnico e histórico. En este sentido la conservadora Cornelia Weyer afirma cómo "la existencia de una gran proporción de material original no es sólo el criterio para que una obra de arte sea original" (Weyer, 2010:21). Al hilo de esta argumentación, en el caso del arte urbano, la originalidad de una obra radica en que conserve la característica de su propia definición de "urbano", es decir que permanezca in situ en el lugar para el que fue realizada.

En cuanto a los diferentes intentos de musealización del arte urbano, ya no se valoraría sólo su arranque mural, sino incluso el de su réplica, como es el caso de la realización de calcos. La obra de Keith Haring Todos juntos podemos parar el sida, que realizó en 1989 en el barrio del Raval de Barcelona, es un claro ejemplo de ello. Este mural, realizado sobre un edificio que posteriormente fue demolido, se ha reproducido usando el calco del original, lo que supone una "copia" de una obra de arte urbano que ha perdido su identidad como auténtica, ha dejado de ser autógrafa para pasar a ser realizada por el equipo de restauradores del Museo de Arte Contemporáneo de Barcelona (MACBA), asumiendo, por parte de todas las partes implicadas, que su identidad como obra radica en la imagen misma que es reproducida. En cuanto a la concepción de reproductibilidad, debemos plantear cómo la imagen estética tiene una evidente notoriedad en la sociedad actual y cómo su reflejo puede también estar contemplado en el arte y en su conservación. Aunque, en el arte urbano, se debe entender más allá de la representación formal que es fijada en un muro, una marquesina o el propio asfalto de la ciudad.

El arte urbano desarrolla su propia identidad a partir de diferentes factores que abarcan desde su contexto y localización, hasta el hecho de ser ilegal y furtivo, por lo que el apropiacionismo desde la institución no deja de ser un proceso forzado que genera la momificación de un arte vivo y callejero, para convertirlo en objeto. Estas obras fueron creadas con una fuerza de expresión y comunicación colectiva y social que, dentro de un museo, carece de significado.

\section{Conclusión}

La conservación del arte urbano presenta una dialéctica entre la estructura material y la conceptual, en la que se han de tener en cuenta una gran cantidad de variables. En cuanto a la estructura material, lleva implícito el estado de conservación, en el que hay que incluir los 
cambios sufridos a lo largo del tiempo. Además de su integridad estética, es importante el reconocimiento del significado intangible de la intervención, el mensaje que transmite y su contexto urbano en la convivencia y diálogo con la colectividad, lo que el filósofo alemán Martín Heidegger (1889-1976) llamaba el world de la obra de arte.

Por ello, para respetar la autenticidad de la creación artística, no sólo hay que tener en cuenta la conservación del material como documento histórico, sino también la obra como creación en su concepto más global, abarcando conceptos como la autenticidad, la intencionalidad, el contexto y el mensaje intelectual.

La restauración no puede limitarse a documentar, fotografiar y geolocalizar el arte que emerge en las medianeras de las ciudades, y aunque no se pueda pretender enmarcarlo dentro de lo que se considera bien patrimonial, se deben valorar diferentes vías de conservación de algunas obras relevantes, siempre basadas en una metodología conservativa fundamentada en la ética de la profesión. La decisión de conservar o no el arte urbano dependerá de la voluntad de diferentes agentes: de los artistas, de una comunidad vecinal y de su propietario, entre otros. En definitiva, de las personas que habitan el espacio circundante y a las que pertenece el paisaje visual, las que conviven y las que, durante un periodo de tiempo determinado, se sentirán identificadas con esas manifestaciones artísticas. Por todo ello, el arte urbano ha de permanecer, en la medida que sea valorado socialmente, como creación potencialmente conservable y restaurable, pero siempre en su contexto original, en la calle, que es el medio donde y para el que las obras fueron creadas.

\section{Notas}

[1] Proceso desarrollado sobre la base de un modelo elaborado de Ernst van De Wetering para el arte tradicional, Modern art, who cares?, Ámsterdam, The Foundation for the conservation of modern art and the Netherlands Institute for Cultural Heritage, 1999.

[2] http://www.bez.es/887119047/instituciones-arte-urbanografiti.html [consulta: 20/09/16]

[3] https://www.incca.org [consulta: 20/09/16]

\section{Bibliografía}

BRANDI, C. (1963). Teoria del Restauro, Einaudi, Turín.

CARBONI, M., (2014). "Tutela, conservazione e restauro dell'arte contemporanea: I'orizonte filosofico". En Tra memoria e oblio. Percorsi nella conservazione dell'arte contemporanea, Roma: Lit Edizioni.
DE DIEGO, Jesús. (2000). Graffiti, la palabra y la imagen: un estudio de la expresión en las culturas urbanas en el fin del siglo XX. Barcelona: Los Libros de la Frontera.

FERNÁNDEZ, B. Lorente, J.P. (2009). Arte en el espacio público: barrios artísticos y revitalización urbana. prensas Universitarias Zaragoza. Zaragoza.

FIGUEROA-SAAVEDRA. F. (2006). Graphitfragen. Una mirada reflexiva sobre el graffiti. Madrid: Minotauro Digital D.L.

GARCÍA GAYO, E. (2011). ¿Se debe conservar el arte urbano basado en la premisa de: "piensa, crea, actúa y olvida"? en: Conservación de Arte Contemporáneo, $12^{\circ}$ Jornada. MNCARS. GE-IIC. Madrid.

HERMAN ABEN, K., (1995). "Conservation of Modern Sculpture at the Stedelijk Museum, Amsterdam. En From Marble to chocolate. The conservation of Modern Sculpture. Edited by Jackie Heuman. Tate Gallery Conference. 18-20 september. Achetype Pbulications Ltd. 105-109.

LLAMAS PACHECO, R. (2010). Conservar y restaurar el arte contemporáneo: un campo abierto a la investigación. Valencia: Universitat Politècnica de València D.L.

MARTINEZ JUSTICIA, J.M., (2000). Historia y Teoría de la Conservación y restauración artística, Madrid, Ed. Tecnos.

MERRILL, S. (2014). "Keeping it real?: subcultural graffiti, street art, heritage and authenticity". International Journal of Heritage Studies: 1-21

RUBIA LÓPEZ, EDL. (2013). Arte urbano: grafiti y postgrafiti. Acercamiento a la problemática legal y patrimonial en torno a su conservación. (http://hdl.handle.net/10251/39193) Máster oficial en CYRBBCC. UPV.

SCHINZEL, H., (2003) "La intención artística y las posibilidades de la restauración", en ALTHÖFER, H., Restauración de pintura contemporánea, tendencias, materiales, técnica, Madrid, Editorial Istmo, 45-64.

SUREDA, J., GUASCH, A.M., (1987) La trama de lo moderno, Madrid, Akal/Arte y estética.

TEMKIN A. Stange Fruit. (1999). En “Corzo, M.A, Mortality immortality? Los Angeles: The Getty conservation institute.

WEYER, C., (2010) "Media art and limits of established ethics of restoration", en SCHÄDLER-SAUB, U., WEBER, C., Theory and practice in the conservation of modern and contemporary art: reflections on the roots and the perspectives: proceedings of the international symposium held. 13-14 January 2009 at the University of Applied Sciences and Arts, Faculty Preservation of Cultural Heritage, Hildesheim, Archetype Publications Ltd. Londres. 


\section{Carlota Santabárbara Morera}

Universidad de Zaragoza

carlotasantabarbara@gmail.com

Doctora en Historia del Arte por la Universidad de Zaragoza y diplomada en conservación y restauración por la Escuela de conservación y restauración de Barcelona. Doctora europea por la Universidad de Zaragoza, con la investigación: “Conservación y restauración del arte contemporáneo. Historia, teoría y crítica". Gestora cultural, graduada por la Universidad Pompeu Fabra de Barcelona.

Colabora como conservadora-restauradora de manera freelance con diferentes instituciones, como el Museo Centro de Arte Reina Sofía de Madrid.

Actualmente es profesora asociada en el Departamento de Historia del Arte de la Universidad de Zaragoza. 